\title{
Fitting of Water Requirement and Yield of Winter Wheat in North China Plain Based on Artificial Neural Network
}

\author{
Weibing Jia, Zhengying Wei*, Lei Zhang, Yubin Zhang, Haoran Wei \\ The State Key Laboratory for Manufacturing Systems Engineering, Xi'an Jiaotong University, Xi'an, China \\ Email: *jiawb02@stu.xjtu.edu.cn
}

How to cite this paper: Jia, W. B., Wei, Z. Y., Zhang, L., Zhang, Y. B., \& Wei, H. R. (2021). Fitting of Water Requirement and Yield of Winter Wheat in North China Plain Based on Artificial Neural Network. Journal of Geoscience and Environment Protection, 9, 21-32.

https://doi.org/10.4236/gep.2021.94003

Received: March 5, 2021

Accepted: April 9, 2021

Published: April 12, 2021

\begin{abstract}
The fitting of water requirement and yield during the growth period of winter wheat can improve yield effectively and improve irrigation water use efficiency with a certain amount of resource input. This paper selects the irrigation amount, precipitation and yield of winter wheat at the Wuqiao Scientific Observation and Experimental Station. Fitting the water requirement and yield of winter wheat based on three types of artificial neural networks. This paper uses support vector machine (SVM), thought evolution algorithm to optimize BP neural network (MAE-BP) and generalized regression neural network (GRNN) to fit the water requirement and yield of two crops. The SVM is the model with the highest fitting accuracy among the three models, the RMSE, MAE, NS and R2 between predictive value and true value are 7.45 $\mathrm{kg} /$ hectares, $213.64 \mathrm{~kg} /$ hectares, $0.8086,0.9409$ respectively.
\end{abstract}

\section{Keywords}

Winter Wheat, Water Requirement, Winter Wheat Yield, Artificial Neural Networks

\section{Introduction}

The North China Plain is important food production areas in China (Zhai, 2017). The effective arable land in this region accounts for up to $21 \%$ of the country's total arable land, but it only accounts for $8 \%$ of the total water resources, including surface and groundwater. It is the most prominent place where the contradiction between the supply and demand of irrigation water in china, and the overexploitation of the groundwater level in the North China 
Plain has made North China the largest low funnel in the world (Zhao, Xiao, Bai, \& Tang, 2020). Winter wheat and maize are the main food crops in the region. The winter wheat planting period is from October last year to June this year, and the maize planting period is from mid-June to late September. The precipitation from June to September accounts for $65 \%$ to $85 \%$ of the annual precipitation, and the precipitation can basically meet the water requirements of each growing period of maize. The average water requirement during the whole growth period of winter wheat is about $450 \mathrm{~mm}$. However, the natural precipitation in the winter wheat growing season in this area can only meet about $30 \%$ of the normal water requirement demand, which cannot meet the healthy growth of winter wheat in North China (Bai, Wan, \& Kang, 2018). The fitting of water requirement and yield during the growth period of winter wheat can increase yield and improve irrigation water use efficiency with a certain amount of resource input.

Relevant scholars have conducted a large number of studies on the water requirement and yield of winter wheat $(\mathrm{Hu}, \mathrm{Ma}, \mathrm{Wu}$, Sun, Feng, Kang, et al., 2020), most of which reflect the water requirements and changes of different crops under high-yield irrigation conditions (Deng, Zhang, Liang, Li, Yang, Wang, et al., 2020). However, a large number of irrigation practices show that the crop itself has physiological water saving and drought resistance capabilities (Yan, Dong, Li, Duan, Yang, Li, et al., 2019). Crop production function is a quantitative description of crop water requirement and yield (Chen, Marek, Marek, Porter, Brauer, \& Srinivasan, 2021), which can determine the economic water demand index of crops scientifically (Wang, Meng, \& Chen, 2019). With the development of artificial intelligence technology in recent years, a series of artificial neural network technologies and algorithms have emerged (Nguyen, Nguyen, Vranova, Nguyen, Bui, \& Khieu, 2021). These technologies and methods can carry out the construction of non-linear relationships and complex logical operations. They are used in prediction, model construction, dynamic simulation and pattern recognition (Fan \& Zhang, 2021). A strong advantage has been exerted in the research. This paper selects the irrigation amount, precipitation and yield of winter wheat at the Wuqiao Scientific Observation and Experimental Station of the Ministry of Agriculture of the Chinese Ministry of Agriculture. Fitting the water requirement and yield of winter wheat based on artificial neural networks, and choose the best method that fits the water requirement and yield of winter wheat.

\section{Material and Methods}

\subsection{Overview of Research Sites}

The Wuqiao Scientific Observation and Experimental Station for Crop Water Efficient Water of the Ministry of Agriculture was selected as the research sites. The experimental station is located in Cangzhou City ( $\left.37^{\circ} 41^{\prime} \mathrm{N}, 116^{\circ} 37^{\prime} \mathrm{E}\right)$, Hebei Province, China. The station belonging to the Heilonggang Basin of the Haihe Plain, average elevation of the station is 14 meters to 23 meters, the groundwater 
level in the station is about $6 \mathrm{~m}$ to 9 meters, the annual precipitation is $550 \mathrm{~mm}$, $64 \%$ of the annual precipitation is concentrated in summer, and the climate type is warm temperate monsoon climate, the annual average temperature of the research site is $12.5^{\circ} \mathrm{C}$. The agricultural planting structure is simple relatively, the main planting crop is winter wheat and maize rotation. The growing season of winter wheat is from October of last year to September of this year, and the growing season of maize is from mid-June to the end of September (Zhao, 2014).

\subsection{Date Acquisition and Overview}

The yield of winter wheat depends on the water requirement and fertilizer in each growth period. The available water for winter wheat in the North China Plain mainly comes from irrigation and natural precipitation. From October of 2013 to June of 2016, there are three complete winter wheat growth periods. The irrigation water treatment of winter wheat was divided into single irrigation mode, two irrigation mode and three irrigation mode. For the above three winter wheat growth periods, the irrigation water volume from the sowing period to the jointing period, the jointing period to the flowering period and the flowering period to the maturity period were counted respectively. A total of 41 sets of irrigation water and yield data were obtained $(\mathrm{Xu}, 2018)$. The experimental data was shown in Figure 1. It can be seen from the figure that the amount of irrigation water from the sowing stage to the jointing stage is the least in winter wheat productive period. The amount of irrigation water from the jointing stage to the flowering stage is greater generally than that from the sowing stage to the jointing stage. At the same time, for 41 different irrigation treatments, the yield of winter wheat ranges from $7000 \mathrm{~kg} /$ hectare to $10,000 \mathrm{~kg} /$ hectare.

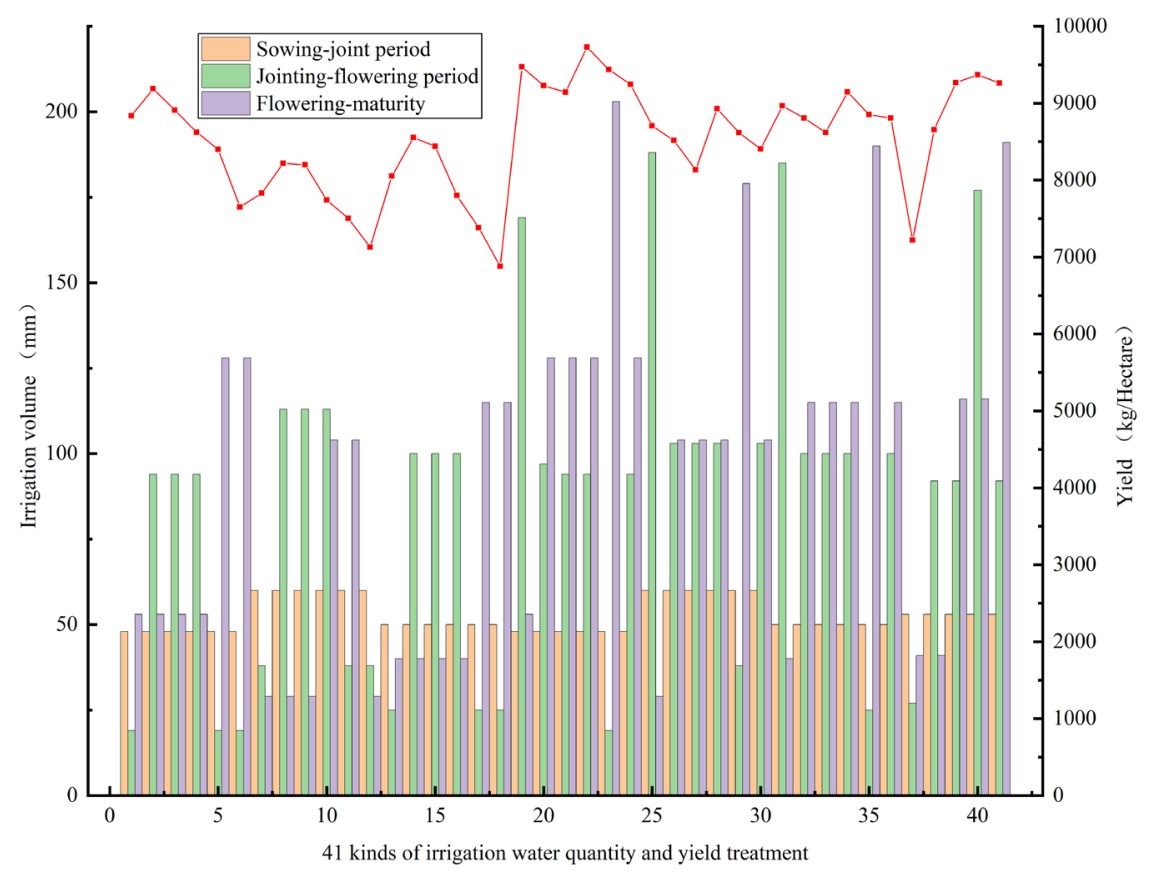

Figure 1. Date of irrigation volume and yield of winter wheat 41 groups. 
Based on meteorological data from China Meteorological Data Network, the precipitation in the research station from October of 2013 to June of 2016 was counted $(\mathrm{Xu}, 2018)$. The precipitation data was shown in Table 1 . The table shows three precipitation of different period of winter wheat. For three growth period, the precipitation between sowing period and jointing period is $19 \mathrm{~mm}$ to $53 \mathrm{~mm}$, the precipitation between jointing period and flowering period is $29 \mathrm{~mm}$ to $60 \mathrm{~mm}$, the precipitation between flowering period and maturity period is 25 $\mathrm{mm}$ to $50 \mathrm{~mm}$. The precipitation between jointing period and flowering period is the most period, the precipitation between sowinging period and jointing period is the minimal period.

\section{Model Establishment}

There are many fitting methods for water requirement and yield of winter wheat. Support vector machine (SVM) can provide good generalization ability, and has the advantages of versatility, robustness, effectiveness, and simple calculation. The characteristic of the BP neural network optimized by the thought evolution algorithm is to divide the group into a winning subgroup and a temporary subgroup, which can memorize multiple generations of information, and can adopt a parallel computing structure. The GRNN network structure is relatively simple, and good results can be obtained by using only cross-validation to optimize the smoothing parameters of the radial basis function used. This paper uses support vector machine (SVM), thought evolution algorithm to optimize BP neural network and generalized regression neural network (GRNN) to fit the water requirement and yield of two crops.

\subsection{Support Vector Machines (SVM)}

From the perspective of linear separable model classification, the main idea of SVM is to select an optimal decision hyperplane to maximize the distance between the two types of samples on both sides of the plane, thereby providing good generalization capabilities for classification problems. When using SVM fit, it was necessary to adjust the relevant parameters, including the penalty factor $\mathrm{c}$ and the kernel function parameter $\mathrm{g}$ (Wang, Zhang, \& Yao, 2021).

In order to obtain an ideal prediction classification accuracy rate, the common method was the cross-validation method. The original data was grouped, one part was used as the training set and the other part was used as the validation set. The training set was used to train the classifier and the validation set.

Table 1. Precipitation in different growth periods.

\begin{tabular}{cccc}
\hline \multirow{2}{*}{ Date } & \multicolumn{3}{c}{ Precipitation $(\mathrm{mm})$} \\
\cline { 2 - 4 } & Sowing -jointing period & Jointing-flowering period & Flowering-maturity \\
\hline $2013-2014$ & 48 & 60 & 50 \\
$2014-2015$ & 19 & 38 & 25 \\
$2015-2016$ & 53 & 29 & 40 \\
\hline
\end{tabular}


The classification accuracy obtained was used as the performance index of the classifier. The group of $\mathrm{c}$ and $\mathrm{g}$ that make the training set verification classification accuracy rate was the best, then the group of $\mathrm{c}$ and $\mathrm{g}$ was selected as the best parameters, and when the classification accuracy rate was the same, the smallest $\mathrm{c}$ was selected first to prevent the over-learning state from occurring, in order to realize the classification of different samples, it is usually necessary to use the classification hyperplane. Equation (1) is the classification hyperplane of support vector machines

$$
f(x)=<w, x>+b=0
$$

Note that $x \in R^{n}, w \in R^{n}, b \in R^{n}, w$ is normal vector of classification hyperplane.

Equation (2) is the corresponding classifier of support vector machines

$$
f(x, w, b)=\operatorname{sgn}(<w, x>+b)
$$

Note that sgn is symbolic function. Overall flow chart of SVM regression model was shown in Figure 2. First select the dependent variable and the effect variable according to the model assumptions, then cross-validation selects the best parameters for regression, then train SVM with the best parameters, the last fit prediction.

\subsection{Generalized Regression Neural Network (GRNN)}

The generalized regression neural network has a strong nonlinear mapping ability, a flexible network structure and a high degree of Fault tolerance and robustness, suitable for solving nonlinear problems. The neural network is suitable for standardizing the detection data. Diagram of generalized regression neural network structure was show in Figure 3.

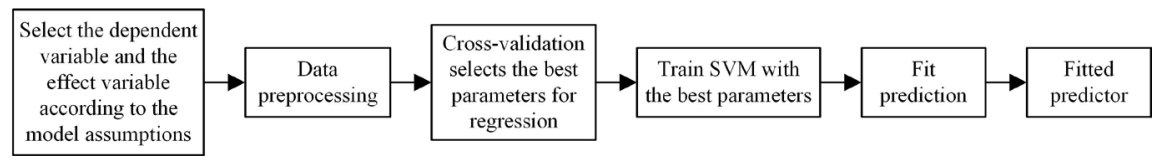

Figure 2. Overall flow chart of SVM regression model.

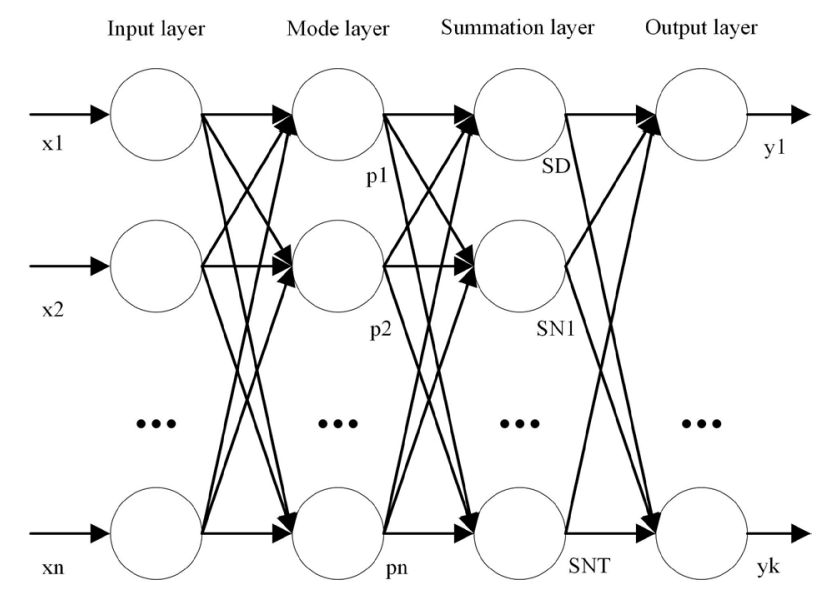

Figure 3. Diagram of generalized regression neural network structure. 
The generalized regression neural network protects the input layer, model layer, summing layer and output layer (Krutikov, Meltsov, Lapitsky, \& Rostovtsev, 2020). The dimension of the input layer is $\mathrm{n}$, and the linear function is the transfer function. The pattern layer is fully connected with the input layer, and there is no connection in the layer. The number of neurons in the pattern layer is equal to the number of samples, that is, $\mathrm{n}$ and the transfer function is a radial basis function. The summation layer outputs the sum of the nodes of each mode layer. The output layer is the final output of the network.

\subsection{Thinking Evolutionary Algorithm Optimizes BP Neural Network (MEA-BP)}

The BP neural network has a multi-layer feedforward neural network, the signal is propagated forward, and the error is transmitted backward. According to the predicted error, the weight and threshold of the network are adjusted in real time, so that the predicted value is constantly close to the expected value. The core of the Mind Evolutionary Algorithm (MEA) is similar to the evolutionary process of human thinking. It is a new type of evolutionary algorithm derived from the genetic algorithm. On the basis of retaining the genetic algorithm, two new types of convergence and alienation are proposed. The operator of through convergence, alienation and other operations, continuous iteration makes the predicted value and the expected value gradually approach (Feng, Wei, Zhang, Zhang, Zhang, \& Jia, 2019).

Evolutionary algorithm optimizes BP neural network was show in Figure 4. After obtaining sample data, the algorithm will generate an initial population, a winning subpopulation, and a temporary subpopulation. The subpopulations perform convergence operations to obtain mature subpopulations, and then perform alienation operations to obtain the overall situation. The optimal individual uses this individual as the weight and threshold for training the BP neural network, so that the BP neural network obtains the predicted value at a faster rate and accuracy.

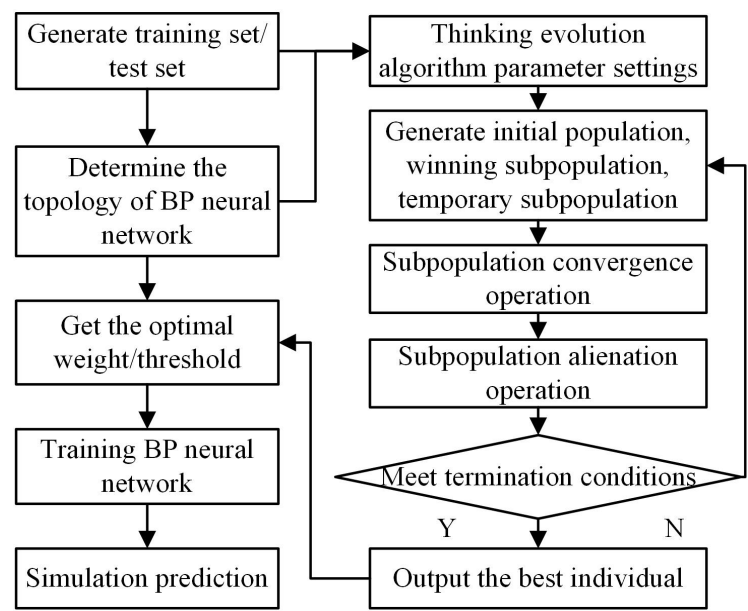

Figure 4. Diagram of evolutionary algorithm optimizes BP neural network structure. 


\section{Results}

To verify the generalization capability of the three different constructed model, Error, Relative Error, Mean Absolute Error (MAE), Root Mean Squared Error (RMSE), Nash-Sutcliffe (NS) and R Squared $\left(\mathrm{R}^{2}\right)$ were selected to indicate the performance of the different models.

\subsection{Fitting Results Based on SVM}

The 29 sets of water requirement and yield data were used for data preprocessing. Through cross-validation, the best parameters of $\mathrm{c}$ and $\mathrm{g}$ were 11.3137 and 0.50 respectively. The best parameter SVM is used for training and fitting prediction. The fitting error and relative error obtained are shown in Figure 5 and Figure 6, respectively. It can be seen from the figure that the change trend of the fitted value and the actual value is basically the same, but there is a sample with a large deviation between the predicted value and the actual value. The maximum error is $851.38 \mathrm{~kg} /$ hectares, the minimal error is $23.15 \mathrm{~kg} / \mathrm{hectares}$, and the maximum relative error is $9.78 \%$, the minimal relative error is $0.25 \%$. The relative error of fitting for $89 \%$ of the samples is controlled within $\pm 5 \%$.

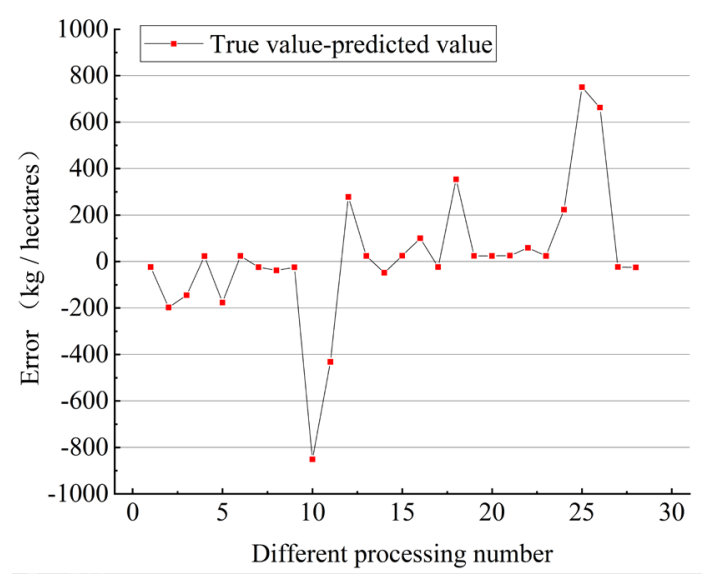

Figure 5. Fitting error based on SVM.

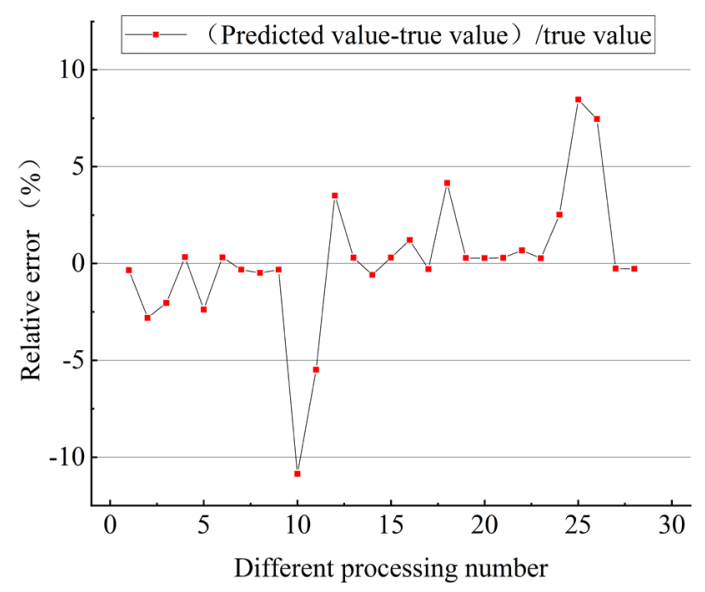

Figure 6. Fitting relative error based on SVM. 


\subsection{Fitting Results Based on GRNN}

The data was preprocessed using 41 sets of water requirement and output test data. Through cross-validation, the best spread value was obtained as 0.1. GRNN was established with the best parameters and fitted predictions were made. By comparison the actual value and the fitted value, the fitting error and relative error of 16 sets of test data were show in Figure 7 and Figure 8. It can be seen from the figure that except for one of the fitted values is smaller than the actual value, the others are larger than the actual value, and the trend of the fitted value and the actual value is basically consistent. The maximum error is 812.50 $\mathrm{kg} /$ hectares, the minimal error is $48.85 \mathrm{~kg} /$ hectares, and the maximum relative error is $10.23 \%$, the minimal relative error is $0.55 \%$. The relative error of fitting for $87.5 \%$ of the samples is controlled within $\pm 5 \%$.

\subsection{Fitting Results Based on MEA-BP}

Optimized the BP neural network based on the thought evolution algorithm, using 25 sets of water requirement and output test data to randomly generate initial populations, cross-convergence operation to obtain the winning subpopulation and temporary subpopulation, obtain the optimal weight or threshold value

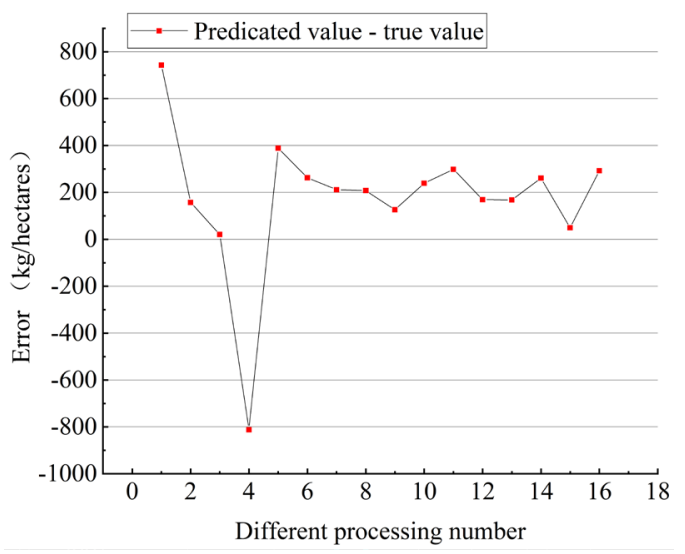

Figure 7. Fitting error based on GRNN.

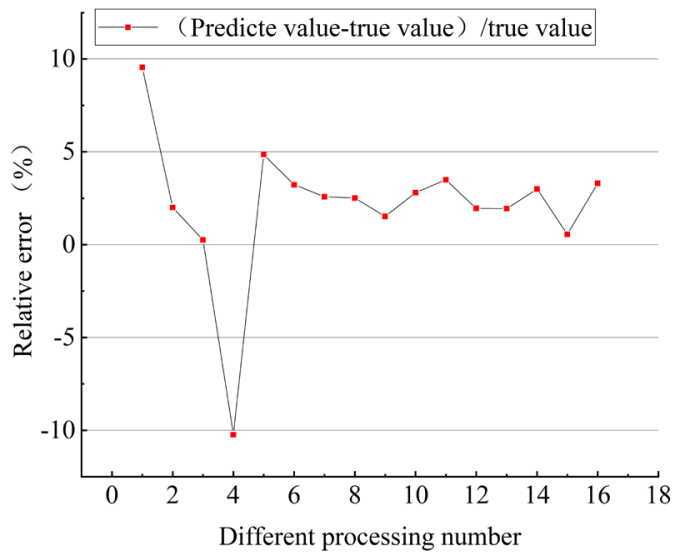

Figure 8. Fitting relative error based on GRNN. 
and perform BP neural network training, and then pass the trained BP neural network, the fitting of 17 sets of water requirement and production was performed.

The error and relative error of the 17 sets of test data fitting and the comparison of the actual value and the fitted value are shown in Figure 9 and Figure 10 respectively. It can be seen from the figure that the change trend between the fitted value and the actual value is similar. The fitted value is generally larger than the actual value, but there are several samples with larger deviations from the fitted value than the actual value. The maximum error value is 1132.54 $\mathrm{kg} /$ hectares, the minimal error is $7.89 \mathrm{~kg} /$ hectares. The maximum relative error is $13.32 \%$, and the minimal relative error is $0.08 \%$. The relative error of fitting for $76.5 \%$ of the samples is controlled within $\pm 5 \%$.

\subsection{Performance Comparison of Different Models}

The results of performance comparison for three artificial neural networks models are shown in Table 2. The RMSE of the predicted and true values of the three models are $7.45 \mathrm{~kg} /$ hectares, $346.21 \mathrm{~kg} /$ hectares and $431.10 \mathrm{~kg} /$ hectares respectively. The MAE of the predicted and true values of the three models are 213.64 $\mathrm{kg} /$ hectares, $346.21 \mathrm{~kg} / \mathrm{hectares}$ and $431.10 \mathrm{~kg} /$ hectares respectively. The NS of predicted and true values of the three models are $0.8086,0.6844$ and 0.5431 respectively. The $\mathrm{R}^{2}$ of predicted and true values of the three models are 0.8086 , 0.6844 and 0.5431 respectively. Consideration of four model evaluation indicators, it can be known that the performance indicators of the SVM algorithm

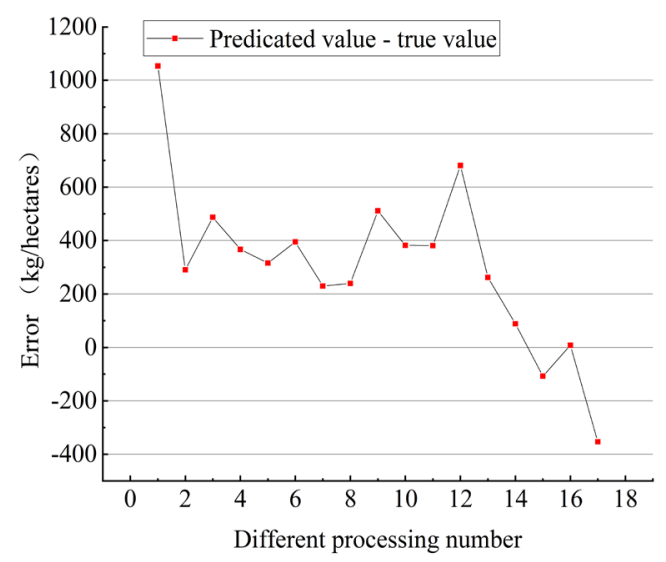

Figure 9. Fitting error based on MAE-BP.

Table 2. Comparison of fitting accuracy of three models.

\begin{tabular}{ccccc}
\hline \multirow{2}{*}{ Model type } & \multicolumn{4}{c}{ Fitting accuracy } \\
\cline { 2 - 5 } & RMSE & MAE & NS & $\mathrm{R}^{2}$ \\
\hline SVM & 7.45 & 213.64 & 0.8086 & 0.9409 \\
GRNN & 346.21 & 275.16 & 0.6844 & 0.7810 \\
MAE-BP & 431.10 & 361.81 & 0.5431 & 0.5600 \\
\hline
\end{tabular}




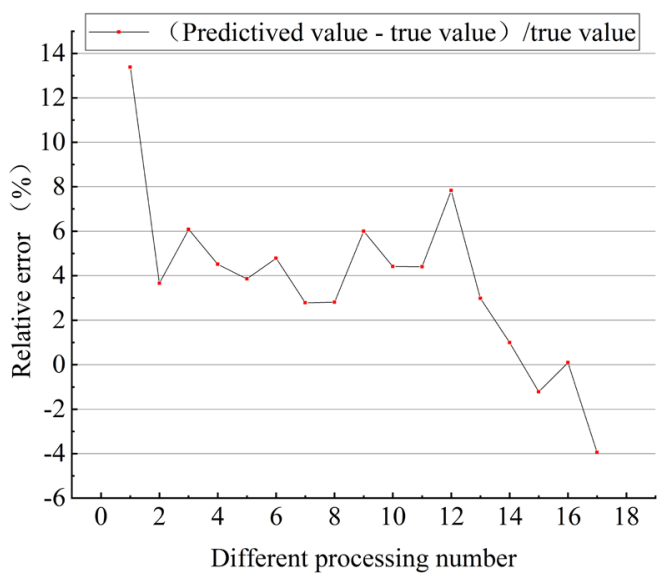

Figure 10. Fitting relative error based on MAE-BP.

model are better than those of GRNN and MAE-BP neural network. SVM model can predict the non-linear relationship between winter wheat water requirement and yield very well.

\section{Conclusion}

This paper takes winter winter wheat from the Wuqiao Scientific Observation and Experiment Station in the North China Plain as the research crop. The winter winter wheat in the experiment station was irrigated once, irrigated twice and irrigated three times throughout the growth periods from October 2013 to June 2016. A total of 41 sets of winter wheat irrigation and yield data were obtained from these experiments. At the same time, A total of 9 sets of precipitation were obtained from the meteorological data. Finally, 41 sets of winter wheat water requirement and yield data were obtained.

The support vector machine, generalized regression neural network and thought evolution algorithm optimize the BP neural network were constructed. Three neural models were used to fit 41 groups of winter wheat water requirement and yield, and the fitting accuracy of the three models was obtained. A comprehensive comparison of the four fitting accuracy indicators shows that the support vector machine is the most suitable model for the winter wheat water requirement and yield fitting of the experimental station.

\section{Acknowledgements}

My deepest gratitude goes first and foremost to Professor Wei Zhengying, my tutor, for her constant encouragement and guidance. This work was supported by the National Key Research and Development Project [grant number 2017YFD0201504-2].

\section{Conflicts of Interest}

The authors declare no conflicts of interest regarding the publication of this paper. 


\section{References}

Bai, S. S., Wan, S. Q., \& Kang, Y. H. (2018). Effects of Drip Fertigation on Winter Wheat Growth and Water Use in North China Plain. Transactions of the Chinese Society for Agricultural Machinery, 49, 269-276.

Chen, Y., Marek, G. W., Marek, T. H., Porter, D. O., Brauer, D. K., \& Srinivasan, R. (2021). Simulating the Effects of Agricultural Production Practices on Water Conservation and Crop Yields Using an Improved SWAT Model in the Texas High Plains, USA. Agricultural Water Management, 244, 1-12. https://doi.org/10.1016/j.agwat.2020.106574

Deng, J. Q., Zhang, Z. X., Liang, Z. T., Li, Z., Yang, X. L., Wang, Z. K., et al. (2020). Replacing Summer Fallow with Annual Forage Improves Crude Protein Productivity and Water Use Efficiency of the Summer Fallow-Winter Wheat Cropping System. Agricultural Water Management, 230, 1-9. https://doi.org/10.1016/j.agwat.2019.105980

Fan, L. Y., \& Zhang, L. A. (2021). Multi-System Fusion Based on Deep Neural Network and Cloud Edge Computing and Its application in Intelligent Manufacturing. Neural Computing and Applications, 1-10. https://doi.org/10.1007/s00521-021-05735-y

Feng, P. C., Wei, Z. Y., Zhang, Y. B., Zhang, Q., Zhang, L., \& Jia, W. B. (2019). Reference Crop Evapotranspiration Prediction for Greenhouse Melon Based on Mind Evolutionary Algorithm Optimization and BP Neural Network. Water Saving Irrigation, 9, 36-39.

Hu, Y. J., Ma, P. H., Wu, S. F., Sun, B. H., Feng, H., Pan, X. L., et al. (2020). Spatial-Temporal Distribution of Winter Wheat (Triticum aestivum L.) Roots and Water Use Efficiency under Ridge-Furrow Dual Mulching. Agricultural Water Management. 240, 1-12. https://doi.org/10.1016/j.agwat.2020.106301

Krutikov, A. K., Meltsov, V. Y., Lapitsky, A. A., \& Rostovtsev, V. S. (2020). EPGA-Implementation of a Prediction Module Based on a Generalized Regression Neural Network. 2020 IEEE Conference of Russian Young Researchers in Electrical and Electronic Engineering (EIConRus).

https://doi.org/10.1109/EIConRus49466.2020.9039414

Nguyen, V. D., Nguyen, H. T. H., Vranova, V., Nguyen, L. T. N., Bui, Q. M., \& Khieu, T. T. (2021). Artificial Neural Network Modeling for Congo Red Adsorption on Microwave-Synthesized Akaganeite Nanoparticles: Optimization, Kinetics, Mechanism, and Thermodynamics. Environmental Science and Pollution Research, 28, 9133-9145. https://doi.org/10.1007/s11356-020-10633-2

Wang, H., Zhang, L., \& Yao, L. X. (2021). Application of Genetic Algorithm Based Support Vector Machine in Selection of New EEG Rhythms for Drowsiness Detection. $E_{X}$ pert Systems with Applications, 171, 1-7. https://doi.org/10.1016/j.eswa.2021.114634

Wang, Z. B., Meng, C. S., Chen, J., \& Chen, F. (2019). Risk Assessment of Crop Production Amid Climate Change Based on the Principle of Maximum Entropy: A Case Study of Winter Wheat Production on the North China Plain. International Journal of Plant Production, 13, 275-284. https://doi.org/10.1007/s42106-019-00053-9

Xu, X. X. (2018). Performance and Coordination of Source and Sink System in Winter Wheat under Water-Saving and High-Yielding Irrigation Patterns (pp. 14-70). Beijing: China Agricultural University Dissertation.

Yan, Q. Y., Dong, F., Li, J. H., Duan, Z. Q., Yang, F., Li, X., et al. (2019). Effects of Maize Straw-Derived Biochar Application on Aoil Temperature, Water Conditions and Growth of Winter Wheat. European Journal of Soil Science, 1-10.

https://doi.org/10.1111/ejss.12863

Zhai, Z. (2017). The Effect and Its Related Mechanism of Plow Pan on Crop Production 
and Environmental Benefit (pp.1-15). Beijing: Chinese Academy of Agricultural Sciences Dissertation.

Zhao, Y. Q., Xiao, D. P., Bai, H. Z., \& Tang, J. Z. (2020). Climate Suitability of Winter Wheat and Summer Maize in the North China Plain. Chinese Journal of Ecology, 39, 2251-2262.

Zhao, Z. G. (2014). Modeling the Critical Nitrogen Concentration Root Distribution of Winter Wheat and Its Eco-Efficiency in North China Plain (pp. 1-20). Beijing: China Agricultural University Dissertation. 